


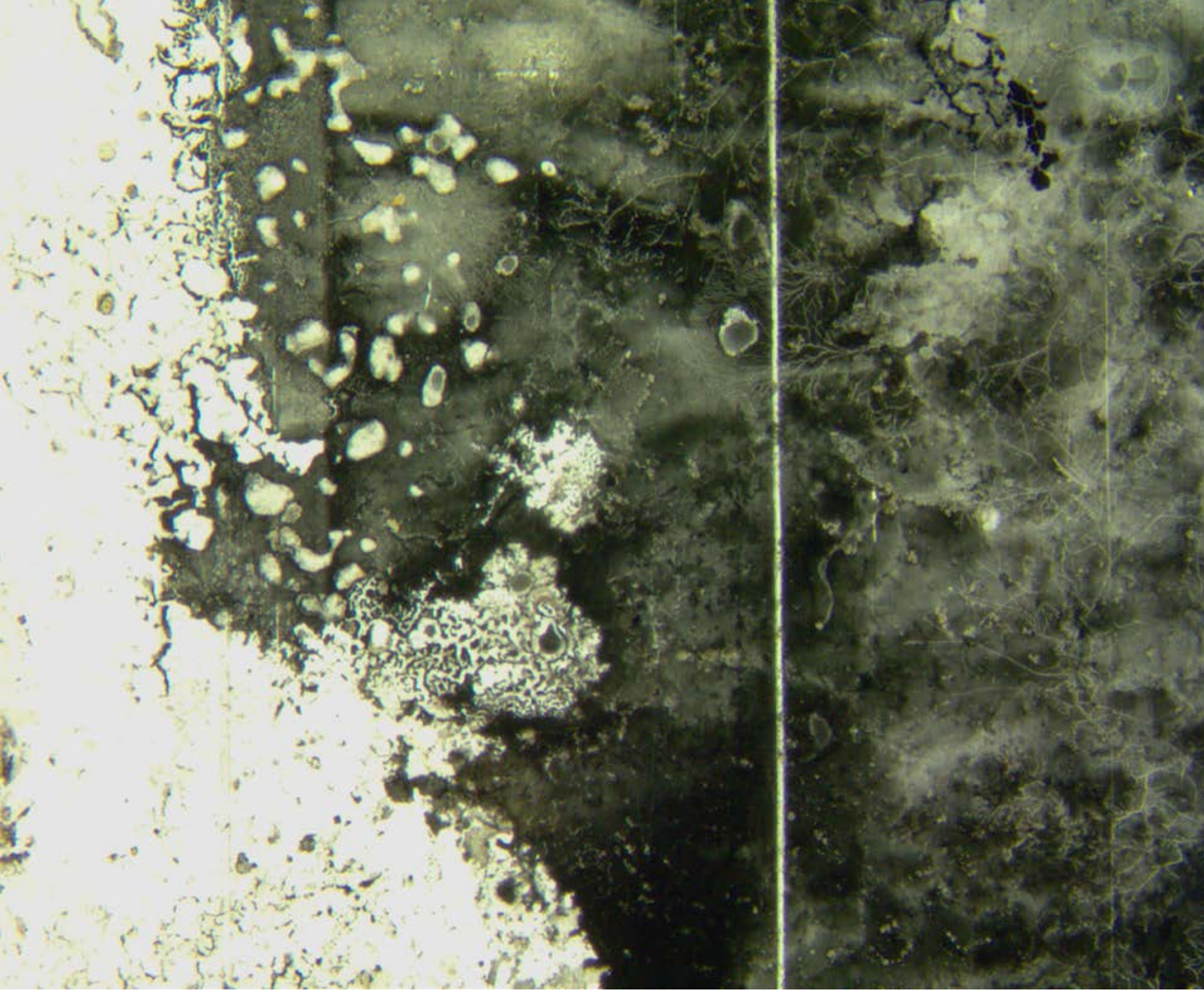

\title{
Políticas de educación superior en Argentina. Entre la COVID-19 y la deuda externa heredada
}

\author{
Estela M. Miranda
}

Doctora en Ciencias de la Educación. Docente-investigadora. Directora de Integración y Conocimiento. Revista del Núcleo de Estudios e Investigaciones en Educación Superior del Mercosur. Universidad Nacional de Córdoba. 


\section{Introducción}

La pandemia y la renegociación de la deuda externa son sin lugar a dudas los dos temas que atraviesan la vida de los argentinos en los últimos meses, cuyos efectos sobre la vida y las instituciones de nuestro pueblo adquieren una magnitud inusitada y una gran incertidumbre. Tanto una como la otra ocupan centralmente la agenda de las políticas públicas del gobierno recientemente asumido. Sin embargo, ambos tienen orígenes y efectos diferentes. La COVID-19 es una pandemia que azota a la humanidad toda, sin atenuantes ni fronteras. En cambio, la abultada deuda externa es apenas una de las consecuencias de las políticas neoliberales del gobierno de la Alianza Cambiemos (2015-2019) que en cuatro años contrajo un escandaloso endeudamiento, generó una alta concentración de la riqueza y "modificó la matriz productiva del país" (Quevedo, 2017, p.12).

En este contexto altamente complejo, las universidades con sus capaci-

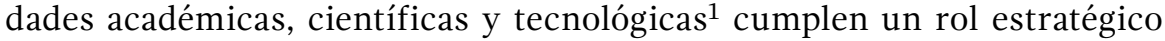
en el asesoramiento, diseño y desarrollo de políticas públicas de salud a los Estados nacionales, provinciales y municipales, la realización de investigaciones para producir insumos y medicamentos, la atención de problemáticas sociales y psico-emocionales, violencia de género y crisis alimentaria, poniendo al servicio de la sociedad todo el capital académico y de conocimiento acumulado en múltiples y variadas prácticas académicas y científicas.

El Ministerio de Ciencia, Tecnología e Innovación de la Nación (MINCYT), conjuntamente con el Consejo Nacional de Investigaciones Científicas y Técnicas (CONICET), la Agencia Nacional de Promoción de la Investigación, el Desarrollo Tecnológico y la Innovación (ANPCYT) y las agencias provinciales de ciencia y tecnología, en definitiva, el sistema científico tecnológico en su conjunto, tan atacado en los cuatro años anteriores por las políticas neoliberales, muestra en esta coyuntura toda la potencialidad acumulada en los institutos de investigación, fundaciones, centros de excelencia, empresas de innovación, instituciones universitarias estatales, provinciales y privadas e institutos de educación superior ${ }^{2}$, en una articulación inédita entre Estado, sistema científico, tecnológico, organizaciones sociales y sectores de la producción.

Las universidades públicas y privadas y los institutos de educación superior desde mediados de marzo de este año mudaron el habitual dictado de clases presenciales al uso intensivo de las tecnologías utilizando las capacidades existentes o readaptándose a las nuevas condiciones, priorizando la continuidad pedagógica para atender una población estudiantil heterogénea y desigual. En las universidades e institutos estatales, con el propósito de atenuar el impacto de la pandemia sobre sectores desprotegidos de la población estudiantil que no cuentan con las condiciones socio-económicas y de conectividad necesarias para adaptarse a esta modalidad extraordinaria de estudios, el gobierno nacional, los gobiernos provinciales, las propias instituciones de educación superior y los Consejos de Rectores desplegaron un conjunto de acciones de mejoramiento de las condiciones materiales y socio-emocionales de contención para garantizar el derecho a la educación superior. Además, se tuvieron que readecuar las 



\section{Consideraciones conceptuales para el análisis de políticas de educación superior}

Tengo el orgullo de haber convocado al Gobierno a numerosos científicos y científicas de Argentina. Somos un Gobierno de científicos, no de CEOs. Un Gobierno con la convicción de que el conocimiento es clave para las políticas públicas y para el desarrollo

(Discurso del Presidente Alberto Fernández, 1 o de marzo de 2020).

Tomo este epígrafe para plantear algunas consideraciones conceptuales acerca del Estado y de las políticas públicas en esta nueva etapa del país. Diferenciándose de la gestión que le antecedió, el gobierno actual propone "un gobierno de científicos" lo que puede ser interpretado como otro modo de hacer políticas públicas. Un importante número de funcionarios del gabinete nacional pertenecen a la carrera de investigador del CONICET y otros son docentes-investigadores de las universidades, corroborando la importancia asignada al conocimiento científico en las políticas públicas y en el desarrollo del país (como analizaremos más adelante).

Al respecto, sabemos que desde hace algunas décadas es recurrente el debate acerca del conocimiento, las ideas y los resultados de las investigaciones en el diseño, ejecución y evaluación de políticas públicas, aunque con marcadas diferencias según las perspectivas y no pocas controversias.

Así, el enfoque de la Policy Science es caracterizado por Brian Fay (citado en Ball, 2011, p. 83) como "conjunto de procedimientos que permiten determinar técnicamente el mejor curso de acción a adoptar para la implementación de una decisión o consecución de objetivos". Se trataría de un tipo de ingeniería política donde el ingeniero político, experto, analista simbólico busca la respuesta técnicamente más adecuada para problemas políticos, de acuerdo con el conocimiento científico socialmente disponible (p. 83).

Se trata de un abordaje tecnocrático para el logro de objetivos políticos y sociales predefinidos que buscan soluciones rápidas, superficiales y, no pocas veces, también legitimar decisiones políticas "utilizando el poder y la autoridad de la ciencia" (Tenti Fanfani,1994, p. 26). El "ingeniero político" aparece por fuera de las estructuras políticas, en ocasiones convocado como especialista/consultor para replicar experiencias exitosas desestimando, por lo general, las condiciones históricas, culturales y sociales de los contextos donde se aplica. Los vínculos con las políticas públicas son contractuales, con mínimos compromisos, mientras circulan entre oficinas gubernamentales locales, nacionales e internacionales con su experiencia para dar soluciones a problemas que otros formularon. En definitiva, se trata de una despolitización y tecnificación de la política por cuanto la previsibilidad del experto queda limitada por las agendas de problemas políticos y sociales definidos de antemano y por otros (Brian Fay en Ball, 2011).

Para el sociólogo inglés Stephen Ball (2011, p. 84) ese tecnicismo solucionador de problemas legitimado con bases científicas "reposa sobre una aceptación acrítica del consenso moral y político que opera dentro de la hegemonía del racionalismo instrumental". En esa misma línea Larner y 
Laurie (citados en Ball, 2017, p. 30) analizan la relación entre el neoliberalismo y expertos o lo que se denomina "tecno-políticos" advirtiendo:

Es crucial reconocer que los diversos expertos que se convierten en "tecnócratas itinerantes", ya sea del norte o del sur global ...no son simplemente formuladores de políticas y expertos técnicos formados en instituciones de élite, sino fanáticos en todo el mundo imponiendo recetas políticas como si la política no existiera y los lugares no importaran ...Los expertos se mueven cada vez más entre las organizaciones privadas, públicas y del tercer sector, y entre las instituciones locales, nacionales e internacionales, y las reconfiguran en consecuencia. (Traducción propia).

Un caso del enfoque de la Policy Science, es la "Nueva gestión pública" (New Public Managment) orientada a transformar el aparato estatal y los modos de hacer políticas públicas, desplazando el paradigma burocrático-weberiano por un modelo posburocrático o gerencial, donde el papel técnico del experto se asocia con las intervenciones del Estado atendiendo criterios de eficiencia, eficacia, calidad, liderazgo y rendición de cuentas (Accountability) (Laval, 2018; Yelicich, 2017; Verger, Curran y Parcerisa, 2015).

Durante el gobierno de la Alianza Cambiemos por primera vez en Argentina se configuró "un Estado gerenciado por los CEOs, los gerentes de las principales multinacionales radicadas en el país". Se trata, al decir de Ranciére, de una "democracia sin demos" o posdemocracia, donde "el pueblo desaparece de la escena política y su rol y peso en la escena política es sustituida por una élite corporativa de clase y tecnocrática, donde la soberanía del pueblo es reemplazada por la soberanía y el poder del mercado" (García Delgado, 2017b, p. 49).

En el nuevo gobierno se reconoce al demos como sujeto y objeto de las políticas públicas, cuando el Presidente refiere a: "desde lo político, nos ocupamos antes que nada de los que han sido olvidados"; "es la hora de definir de qué lado va a estar cada uno de nosotros. Nosotros estamos del lado del pueblo"; o convoca a la recuperación de un Estado debilitado, donde casi desaparecieron los instrumentos de regulación, para generar otra construcción diferente: "La idea de un Nuevo Contrato de Ciudadanía Social (que) supone unir voluntades y articular al Estado con las fuerzas políticas, los sectores productivos, las confederaciones de trabajadores, los movimientos sociales, que incluyen el feminismo, la juventud, el ambientalismo". En esa articulación también incorpora al sistema científico-tecnológico y a los sectores académicos (Discurso 01/03/2020).

Desde ese marco entendemos a la política como un proceso social, relacional, temporal, discursivo, desordenado, antes que "como un proceso hiperracional o lineal al "final" de cual encontramos "fallas de implementación" (Ball, 2011, p. 176-177). Uno u otro posicionamiento definen la orientación de la política pública: Si se orienta por criterios tecnocráticos de "eficiencia" o por principios democráticos de "justicia social". 


\section{Revisando el pasado reciente en las políticas de educación superior para saber de dónde venimos y dónde estamos}

El nuevo siglo se inicia en un contexto político latinoamericano con gobiernos de signos nacional-populares, neo-desarrollistas y de centro-izquierda (Bolivia, Venezuela, Ecuador, Argentina, Brasil) y gobiernos neoliberales (México, Colombia, Perú, Chile). A lo largo de la última década ese escenario se fue reconfigurando de otro modo, reafirmando lo que parece ser una constante: el movimiento pendular de las democracias latinoamericanas en una alternancia de gobiernos progresistas y otros promercado o de una derecha conservadora, autoritaria y neoliberal. A diferencia del pasado que lo hacia por golpes de Estado ahora la derecha llega al gobierno con partido propio y por elecciones democráticas (García Delgado, 2017a, 2017b), aunque también por prácticas destituyentes de gobiernos en la "cruzada contra la corrupción".

En Argentina, después de una década de políticas neoliberales que culminaron con la crisis de 2001, los gobiernos nacionales de Néstor Kirchner (2003-2007) y los dos mandatos de Cristina Fernández de Kirchner (20072015) recuperaron la principalidad del Estado como promotor y regulador de las políticas educativas generando un escenario de gobernabilidad y diferenciandose de los discursos y prácticas neoliberales de los años noventa (Chiroleu y Iazzetta, 2005; Suasnábar y Rovelli, 2012, Miranda, 2018).

Las políticas de educación superior sustituyeron la visión mercantilista por nuevos lineamientos que operaron desde una concepción de la educación superior como un bien público social, un derecho humano universal y un deber del Estado, atendiendo a la Conferencia Regional de Educación Superior de 2008 (IESALC, 2008). En palabras de Pérez Rasetti (2014, p. 24) "se explicita un compromiso del Estado con el sostenimiento y el desarrollo de la educación superior, reforzado por un compromiso similar con el desarrollo científico y tecnológico que impacta en las universidades", como una constante que se mantuvo en todos los gobiernos de periodo.

El derecho a la educación superior, en tanto bien público, social y un deber del Estado, transversalizó las políticas y las acciones atendiendo a tres propósitos: inclusión, calidad y jerarquización de la ciencia y la tecnología ${ }^{4}$.

Las políticas de inclusión social y educativa estuvieron orientadas a mejorar las condiciones de acceso, permanencia y finalización de estudios universitarios de los grupos sociales más desfavorecidos. Entre 2004 y 2015 , la matrícula universitaria creció $26.3 \%$ y los egresados $48.6 \%$. Del total de la matrícula estudiantil $57.6 \%$ son mujeres y entre los egresados las mujeres representan 60.7\% (Anuarios Estadísticos, 2005; 2015).

En 2015 la Tasa Bruta de Escolarización universitaria alcanzó 38.6\%, la Tasa Neta de Escolarización Universitaria 19,4\% y la Tasa Bruta de Escolarización de Educación Superior 75.6\% ${ }^{5}$ (SITEAL, 2015; Miranda, 2014). Las instituciones universitarias estatales concentran $74 \%$ de la matrícula, mientras que las instituciones de gestión privada $26 \%$.

También se destaca el crecimiento de la matrícula en los Institutos de Educación Superior (IES) y la alta composición femenina entre el estudiantado. La matrícula creció en diez años (2005-2015) 56.7\% y las estudiantes mujeres 
representan 69.7\% (Anuarios Estadísticos, 2005; 2015; García de Fanelli, 2016).

La expansión del acceso estuvo acompañada por un aumento del gasto público universitario, programas de becas y de ampliación de la oferta institucional atendiendo el fortalecimiento de la distribución territorial de la educación superior universitaria.

Mientras en los noventa el promedio de gasto público universitario fue de $0.56 \%$, del PBI, en la década del 2000 este valor se elevó ligeramente $0.59 \%$ del PBI hasta alcanzar en 2015 0,85\% del PBI. El Gasto público universitario por alumno (2018), aumentó de $\$ 28,955$ (2003) a $\$ 57,559(2015)^{6}$ (Doberti, 2020).

El presupuesto destinado a becas universitarias, entre 2002 y 2010 , pasó de $\$ 7,200,000$ a $\$ 45,063,000$, lo que significó un aumento de $650 \%$ en ocho años. En 2008 se crearon dos nuevos tipos de becas: Programa Nacional de Becas Bicentenario (PNBB) y el Programa Nacional de Becas de Grado TICs (PNBTICs) por lo que el monto del presupuesto destinado a becas universitarias alcanzó los $\$ 320,777,022$. El mayor presupuesto se tradujo en un aumento de las becas universitarias: de 2,726 becas otorgadas (2003) se estiman en más de 50,000 para el año 2013 (Revista Bicentenario, en Miranda, 2013).

El Programa de Expansión de la distribución territorial de la oferta de educación superior universitaria (PROEDESUP) habilitó en el periodo 20032015 la creación de 24 instituciones de gestión estatal y 13 de gestión privada, alcanzando un total de 132 instituciones (Siguiente tabla). Atendiendo a un criterio de distribución territorial y priorizando las provincias o regiones del país que concentran una población con más restricciones socioeconómicas y menor acceso a bienes culturales, las universidades nacionales se ubicaron mayormente en el conurbano bonaerense ${ }^{7}$, en zonas muy pobres "y orientadas a una población estudiantil que hasta entonces había tenido pocas posibilidades de acceder a los estudios superiores" (Donini y Gorostiaga, 2016; Del Valle, Montero y Mauro, 2017; Otero y Otras, 2018; Miranda, 2018).

Cantidad de instituciones universitarias, según régimen

\begin{tabular}{|l|c|c|c|}
\hline \multirow{2}{*}{\multicolumn{1}{|c|}{ Régimen }} & \multicolumn{2}{c|}{ Instituciones } \\
\cline { 2 - 4 } & $\begin{array}{c}\text { Institutos } \\
\text { universitarios }\end{array}$ & Universidades & Total \\
\hline Total & 22 & 110 & 132 \\
\hline Estatal-Nacional & 7 & 55 & 62 \\
\hline Estatal-Provincial & 1 & 5 & 6 \\
\hline Privado & 13 & 49 & 62 \\
\hline Extranjera/internacional & 1 & 1 & 2 \\
\hline
\end{tabular}

Fuente: Departamento de Información Universitaria, DNPEIU-SPU.

En los Institutos de Formación Superior la mayor expansión institucional se produjo en el sector estatal con 75 nuevas creaciones y nueve en el sector privado, totalizando, en 2018, 2,739 instituciones, de las cuales 
$47.8 \%$ son de gestión estatal y $52.2 \%$ de gestión privada. Con relación al tipo de formación ofrecida, $26.1 \%$ se orienta exclusivamente a la formación docente; $41 \%$ exclusivamente a la formación técnico-profesional y 33\% de las instituciones concentran ambos tipos de formación (Anuario Estadístico, 2018; García de Fanelli, 2016).

El Programa de Calidad Universitaria fue diseñado para fortalecer las carreras prioritarias para el desarrollo del país ${ }^{8}$ y reducir la desarticulación entre los distintos sectores del nivel superior y con otros niveles del sistema educativo. En ese marco, la calidad de la educación universitaria deja de ser pensada desde parámetros economicistas de eficiencia, como calidad isomórfica o calidad del modelo único, que vinculaba la formación con la empleabilidad con foco en el profesional competitivo imperante en los años noventa (Morosini, 2017, p. 387). Desde una nueva concepción, la calidad y evaluación universitaria se vincula con la pertinencia social y política, esto es, el mejoramiento de la enseñanza universitaria, el fortalecimiento de las condiciones institucionales, en particular de las nuevas universidades creadas en geografías que albergan un alto porcentaje de grupos sociales vulnerables y la articulación e integración del sistema educativo entre niveles e instituciones.

Entre las líneas de acción del Programa de Calidad Universitaria se destacan los Proyectos para el Mejoramiento de la Enseñanza Universitaria de carreras reguladas por el Estado ${ }^{9}$ destinados a financiar el mejoramiento de la enseñanza en las carreras de grados que cumplimentaron los procesos de acreditación de la Comisión Nacional de Evaluación y Acreditación Universitaria (CONEAU). El Programa de Mejoramiento de la Enseñanza en Ingeniería (PROMEI I y II) fue una de las líneas prioritarias del gobierno nacional por tratarse de "un campo profesional clave para el desarrollo económico nacional"10 (Pérez Rasetti, 2004; Ministerio de Educación, 2005, 2007, 2010; Dibbern, 2010; García de Fanelli y Claverie, 2013).

\section{Compromiso con el desarrollo científico, tecnológico}

Durante los gobiernos de 2003 a 2015, se "retomó el concepto de impulsar el desarrollo económico con base en el $I+D$ local y la expansión del mercado interno y regional. En el discurso político se aludió consistentemente a la intención de transformar la economía argentina hacia una "economía basada en el conocimiento" (Stefani, 2018, p. 8).

Uno de los hitos importantes fue la creación del Ministerio de Ciencia y Tecnología en 2007. Sobre la inversión destinada a las universidades, al desarrollo científico y tecnológico y al fortalecimiento del CONICET, entre 2003 y 2013 la inversión $I+D$ aumentó del $0.49 \%$ del PBI al $0.60 \%$. Sobre la composición del gasto correspondiente al año 2013 por sectores de ejecución de este gasto, García de Fanellí (2016, p.7) observa que las universidades estatales ejecutan $29 \%$ y las privadas $1.5 \%$.

El CONICET que en 2003 contaba con 3.500 investigadores y 2.200 becarios registró, en 2015, 9,200 investigadores, más de 10,000 becarios y 1,335 investigadores repatriados a través del Programa Raíces (Red de Argentinos Investigadores y Científicos en el exterior) (Filmus, 2019, p.7). 


\section{Las políticas de educación superior de la Alianza Cambiemos (2015-2019). En cuatro años tierra arrasada ${ }^{11}$}

El ciclo que se abre a partir del gobierno de la Alianza Cambiemos (20152019) definido como "neoliberalismo tardío" o "neoliberalismo empresarial autoritario" se caracterizó por formas de hacer políticas "posmodernas" y de emprendedurismo. Además, agrega García Delgado (2017a, p. 18), "la retórica anticorrupción contacta con esta forma pospolítica de construcción de sentidos que sostiene el discurso comunicacional distintivo de estos gobiernos neoliberales, pero tardíos". Se trata de un Estado de CEOs, una gobernanza de gerentes que vienen del campo empresarial y pasan a ocupar cargos en la gestión estatal o privatizando las políticas públicas a través de la contratación de consultorías y de think tanks de agencias privadas nacionales y extranjeras para la formulación y ejecución de las políticas (García Delgado, 2017b).

Con un fuerte cuestionamiento al Estado y de desvalorización de la escuela pública el gobierno de Macri apeló a la negación, desconocimiento y desmantelamiento de lo realizado por el gobierno anterior, usando instrumentos de políticas educativas orientados a la eficiencia y a la rendición de cuentas. En el discurso de los funcionarios y con la complicidad de los medios de comunicación concentrados, los problemas educativos son interpretados desde una lógica hiperracional que neutraliza las políticas educativas para reducirlas a cuestiones técnicas que se resuelven usando elementos de gerenciamiento (control y medición de resultados).

Sobre la educación superior se reedita el discurso neoliberal de desprestigio de la universidad pública, gratuita y el ingreso irrestricto, asociando la baja calidad con masividad y deslegitimando su relevancia como dispositivo de movilidad social ascendente y progreso individual y colectivo. En una clara alusión a las universidades estatales creadas en el conurbano bonaerense durante la presidencia de Cristina F. de Kirchner, la gobernadora de la provincia de Buenos Aires se lamentaba: "¿para qué llenar de universidades públicas toda la provincia cuando todos sabemos que nadie que nace en la pobreza llega a la universidad?" (El País Digital, 30/05/18 citado en Miranda, 2020; Página 12, 19/12/2019).

La meta de $6 \%$ de inversión anual del PBI en educación establecido en la Ley de Educación Nacional (2006) no se cumplió en ninguno de los cuatro años de la gestión de la Alianza Cambiemos: Se pasó de $6.1 \%$ del PBI en 2015 a 5.7\% en 2017, previéndose una disminución para el periodo siguiente como resultado del acuerdo firmado con el Fondo Monetario Internacional y los compromisos de recortes del gasto público (IEC-CONADU, 2019; Morduchowicz, 2019).

En los cuatro años, la inversión pública en el sistema universitario cayó un $22.3 \%$ con relación al PIB desde 2015 (Filmus, 2019, p. 8; Doberti, 2020). El Informe elaborado por el IEC-CONADU (2019) sostiene como causas de la caída el retraso del presupuesto universitario frente al proceso inflacionario, la subejecución de las partidas disponibles así como la reducción del financiamiento a las universidades provenientes de convenios con organismos públicos. Además, una disminución abrupta del presupuesto destinado a planes de desarrollo universitario e infraestructura y la eliminación de 


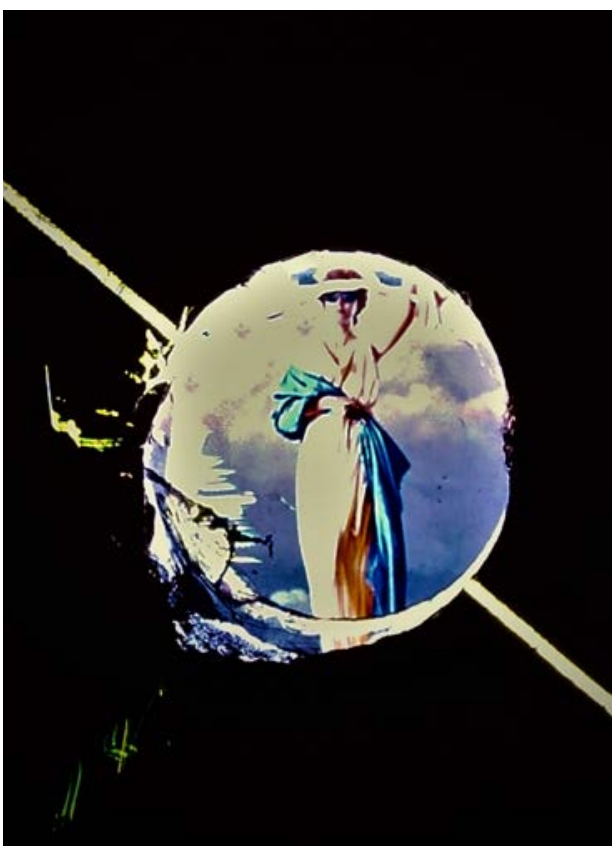

otras fuentes de financiamiento como los programas específicos de la Secretaría de Políticas Universitarias orientados al desarrollo de políticas académicas como el programa de calidad, programa voluntariado, programas de internacionalización y otros proyectos de formación de posgrados y mejoramiento de la enseñanza. (IEC-CONADU, 2019; Página 12, 19/12/2019).

Las becas a estudiantes universitarios, tantos las dirigidas a las carreras prioritarias como a estudiantes de bajos recursos sufrieron también una reducción significativa o en otros casos, como el plan PROGRESAR, se desplazó su sentido solidario hacia a uno meritocrático ${ }^{12}$ (Página 12, 19/12/2019; Aliaga,

2018). El Informe de IEC-CONADU (2019) estimó para 2019 que el valor real del presupuesto destinado a becas universitarias disminuyó $65 \%$, respecto a 2015.

En la gestión del Presidente Macri,

las políticas hacia la ciencia y la tecnología no fueron ajenas a las lógicas del programa de gobierno de Cambiemos; programa que se desentendió del desarrollo industrial y de la capacidad de agregar valor a partir del trabajo y de la aplicación de los resultados de la investigación científica a la modernización de los procesos productivos (Filmus, 2019, p. 3).

Aún cuando a poco de asumir la Alianza Cambiemos había prometido mantener lo realizado en el área por el gobierno anterior, lo que efectivamente hizo fue frenar el cumplimiento de los objetivos propuestos en el Plan Argentina Innovadora 2020 y desarticular los principales programas tecnológicos estratégicos, algunos que venían de varias décadas atrás y otros impulsados por el gobierno anterior, afectando, particularmente, los proyectos que apuntaban a fortalecer la soberanía tecnológica ${ }^{13}$.

El presupuesto para la función Ciencia y Técnica cae $38 \%$, respecto a 2015. (Stefani, 2018; Aliaga, 2018).

En el CONICET se redujo el ingreso a becarios y a la carrera de investigador científico, sobre todo en las áreas de las ciencias sociales, mientras todas las disciplinas pusieron en duda la rigurosidad de la evaluación para el ingreso al CONICET como justificativo de los recortes presupuestarios. El número de nuevos becarios cayó de 1.800 en el 2015 a 1.234 en 2017, mientras el número de ingresos a la carrera de investigador cayó de 943 ingresos en 2015 a 450 en 2019 (Aliaga, 2019). Además, como señala Daniel Filmus 
(2019) "el poder adquisitivo de los salarios y las becas de los científicos se ha reducido fuertemente, en los últimos tres años a valores constantes. El valor del sueldo cayó más de 30\%” (p.10).

\section{Lineamientos políticos del nuevo gobierno para la educación superior: Entre las expectativas sociales y las condiciones objetivas}

El escaso tiempo que media entre la asunción del nuevo gobierno nacional y la irrupción de la pandemia, en medio de la dura negociación de la deuda externa, nos interpela a una presentación provisoria de los lineamientos de una agenda en construcción sobre la educación superior. Como señalara anteriormente, lo que se observa es un consenso generalizado sobre la recuperación y el fortalecimiento de las políticas y las acciones anteriores a los últimos cuatro años de neoliberalismo tardío, atendiendo a los nuevos y muy complejos escenarios nacionales e internacionales.

Asumimos que en el discurso de apertura de las sesiones ordinarias del Congreso Nacional, el Presidente Fernández expuso los lineamientos generales de la agenda de gobierno, preanunciados en la plataforma electoral y refrendados en el discurso de asunción a la Presidencia.

El nuevo gobierno recibía el país en una situación altamente compleja en lo socioeconómico y político: "La Nación está endeudada, con un manto de inestabilidad que desecha cualquier posibilidad de desarrollo y que deja al país rehén de los mercados financieros internacionales" (Discurso 10/12/2019). Durante los primeros 40 meses del gobierno de la Alianza Cambiemos la deuda pública creció en 113,000 millones de dólares, de los cuales casi dos tercios, 68,000 millones, tuvieron como destino la formación de activos externos, es decir, la fuga de capitales. Un país sumergido en una recesión profunda. El índice de inflación durante el año 2019 alcanzó 53.8\%; la desocupación en cuatro años se elevó $9.7 \%$; la pérdida de alrededor de 240,000 empleos privados y una fuerte caída de la actividad industrial que dejó una capacidad ociosa del 40\% (El Cronista, 2019). El Estado nacional registra una deuda en materia de obras públicas de más de 35.000 millones de pesos y $60 \%$ de las obras iniciadas se encuentran paralizadas. Los aumentos tarifarios de los servicios públicos en los cuatro años registraron incremen- 
tos excesivos: El servicio de gas se incrementó en ese periodo alrededor del $2,000 \%$ y el de electricidad cerca del 3,000\%, impactando fuertemente en las familias, comercios, industrias y pequeñas y medianas empresas (Discurso de apertura de las sesiones ordinarias del Congreso Nacional, 1/3/2020; El Cronista, 2019).

En ese contexto, el nuevo gobierno convocaba a la recuperación del rol principal del Estado en educación para "revertir el actual proceso de reducción de la responsabilidad del Estado nacional en materia educativa y la delegación de responsabilidades educativas en organizaciones con fines de lucro" (Plataforma electoral, 2019). Para ello propone aumentar la inversión en educación digital, extensión de la jornada escolar, educación técnica, formación docente, expansión del nivel inicial, infraestructura y equipamiento; también el presupuesto real destinado a universidades y ciencia y tecnología"15 (Redacción, 2019).

Los lineamientos de la agenda en construcción sobre la educación superior refieren a:

- Expansión del sistema universitario. En una línea de continuidad con lo que fueron las políticas de creación de universidades de los gobiernos Kirchneristas y reafirmando el derecho a la educación superior, el nuevo gobierno se plantea:

\begin{abstract}
Recuperar la inversión y las políticas de expansión del sistema universitario en el marco de una planificación seria que signifique seguir ampliando el derecho de todos/as nuestros jóvenes a continuar estudios superiores y a mejorar sus trayectorias y condiciones de graduación y, al mismo tiempo, a dar respuesta a las necesidades de desarrollo nacional a través de la formación de los profesionales que la Argentina necesita y de la producción de conocimiento científico tecnológico que aporte a un desarrollo autónomo y soberano. (Lo resaltado es nuestro).
\end{abstract}

Para lo cual propone:

Una mayor articulación de las políticas universitarias con todos los sectores de la política pública, la producción de información y la formulación de proyecciones sobre los requerimientos científico-tecnológicos y de formación profesional en el mediano y largo plazo, la generación de nuevos perfiles profesionales, la ampliación de nuevas formas de acceso a la formación superior en todos sus niveles a través de medios digitales.

- Jerarquización del sistema científico y tecnológico. En la plataforma electoral del candidato del Frente de Todos, el capítulo de Ciencia y Tecnología ocupa una extensión que evidencia la prioridad asignada a uno de los sectores que había sufrido un profundo deterioro en el gobierno anterior. Ciencia, tecnología e innovación son áreas estratégicas en el proyecto de desarrollo nacional soberano y autónomo. Esa propuesta electoral fue ratificada en el Discurso de asunción a la Presidencia (10/12/2019) al señalar: 
Reivindicamos a la investigación científica y tecnológica porque ningún país podrá desarrollarse sin generar conocimientos y sin facilitar el acceso de toda la sociedad al conocimiento. He decidido que en nuestro Gobierno el área respectiva recupere su jerarquía Ministerial que nunca debió perder.

Una de las primeras medidas fue restableciendo el Ministerio de Ciencia, Tecnología e Innovación, creado en 2007, y que durante el gobierno de la Alianza Cambiemos fue degradado a Secretaría de Ciencia y Tecnología. Para potenciar la investigación científica se amplían los ingresos (becarios de doctorados e ingresos a carrera de investigador) al CONICET y se otorga un aumento en los montos de las becas y salarios de los investigadores.

- El fortalecimiento del vinculo Universidad, comunidad y sector productivo para el desarrollo territorial. En cinco meses desde que asumió el gobierno nacional la articulación de las políticas universitarias y científico-tecnológicas con otros sectores de las políticas públicas se pone en evidencia en esta coyuntura de emergencia sanitaria. El Consejo Interuniversitario Nacional (CIN) destacaba el rol proactivo de las universidades:

En articulación con el Ministerio de Salud, el Ministerio de Educación, el Ministerio de Ciencia, Tecnología e Innovación y los ministerios provinciales, así también, con los gobiernos locales, mediante el desarrollo de distintas acciones como partícipes indispensables de las políticas públicas (...) En ese marco, cada universidad contribuye con la generación y transferencia de conocimiento para el desarrollo de elementos de diagnóstico, preventivos y paliativos (...) Otras instituciones afectan sus laboratorios para el testeo PCR, la disponibilidad de vehículos oficiales, sus edificios para futuras internaciones o aislaciones o para la distribución de ayuda social, sus capacidades tecnológicas para las comunicaciones y campañas informativas, entre otros ejemplos. También, han convocado voluntarios de distintas carreras y disciplinas para desarrollar su pericia en estos campos, e incluso, otros que surjan a partir de los requerimientos propios de esta situación de emergencia sanitaria sin precedentes en nuestro país (...) (CIN, abril de 2020).

\section{Una nueva Ley de Educación Superior}

Proponemos iniciar el debate de una nueva Ley de Educación Superior que permita fortalecer nuestro sistema universitario. La gratuidad y el acceso irrestricto a la universidad son el punto de partida. Fortalecer el vínculo de la universidad con la comunidad y el sector productivo es central para garantizar el desarrollo territorial (Discurso 1/03/2020).

Los debates sobre la necesidad de una nueva ley de educación superior se plantearon en varios momentos y desde diferentes sectores en las últimas décadas. No fue una cuestión sencilla instalar el tema en la agenda pública, 
aún cuando la controvertida Ley de Educación Superior (Núm. 24.125), sancionada en 1995, requería cambios. Con el propósito de atenuar posibles avances sobre derechos adquiridos y de fuerte tradición en la universidad argentina, el Proyecto de Ley presentado al Congreso por la Diputada Adriana Puiggrós habilitó la modificación de algunos artículos y el agregado de otros en la Ley de Implementación efectiva de la responsabilidad del Estado en el nivel de Educación superior, sancionada en octubre de 2015. Dos cuestiones se pueden destacar en la urgencia de esas modificaciones, por un lado efectivizar la gratuidad de los estudios de grado en las instituciones estatales, recordemos que la les habilitaba el arancelamiento de los estudios y, por otro, asegurar la modalidad de ingreso irrestricto a la educación superior. Ambas cuestiones han marcado la historia de la universidad argentina desde la Reforma de 1918 a la actualidad. Si bien formaron parte de los reclamos de los estudiantes en aquella primera gesta universitaria, recién en $1949^{15}$ se suprimieron los aranceles universitarios. Con la recuperación de las instituciones de la democracia en diciembre de 1983 el gobierno de Raúl R. Alfonsín suprimió los exámenes eliminatorios en el ingreso a las universidades y los cupos (numerus clausus), impuestos durante la dictadura militar (1976-1983).

Además, del ingreso, la gratuidad y tantos otros temas a incluir en el tratamiento de una nueva ley, será central el debate sobre "el aporte de las universidades al desarrollo científico-tecnológico autónomo y a los proyectos productivos nacionales y regionales" (Filmus, 2019, p. 11; CRES 2018).

\section{Para seguir pensando. Entre expectativas y desafíos}

El Presidente Alberto Fernández desde la campaña electoral de 2019 había planteado la necesidad de modificar la matriz productiva heredada, que el Gobierno de Cambiemos sostuvo sobre "la esperanza" de la inversión extranjera que nunca llegó salvo para hacer negocios financieros, por un modelo industrial en el que la inversión en ciencia y tecnología es estratégica para un proyecto de desarrollo económico sustentable.

Parafraseando el título de la columnista especializada Nora Bär en un periódico nacional, a pocos días de asumir el nuevo gobierno ¿Qué esperan los científicos y las universidades del nuevo gobierno? Hay una amplia coincidencia en que es urgente superar el retraso para tener un nivel competitivo, pero también en que es necesario acoplar el sistema científico y tecnológico con la actividad productiva y otros sectores sociales para comenzar el sendero de un desarrollo económico sustentable apostando a la incorporación de I+D (La Nación, 2019; Stefani, 2018).

A partir de marzo cuando se detectó el primer caso de $\mathrm{CO}-$ VID-19 en el país entramos en una dimensión desconocida que 

1. En la última década, las universidades han tenido un papel preponderante en la expansión de la actividad científico-tecnológica en América Latina y el Caribe (ALYC). Se estima que $62 \%$ de los investigadores de ALYC se desempeña en universidades, mientras en Europa ese índice es inferior a 40\%. La inversión en educación superior tuvo un aumento en términos reales de $41 \%$ entre 2010 y 2015. En Argentina, más de $70 \%$ de los investigadores de CONICET y 74\% de los becarios doctorales tenían como lugar de trabajo las universidades nacionales o en institutos de CONICET con contraparte en universidades (Del Bello, 2015; Informe OCTS-OEI, 2018).

2. Argentina posee una estructura de educación superior de tipo binaria, conformada por Institutos de Educación Superior (institutos de formación docente, técnico-profesional o artística) (anteriormente denominado no-universitario, de jurisdicción en las provincias y el Gobierno de la Ciudad de Buenos Aires) e instituciones universitarias (universidades e institutos universitarios, autónomos y con jurisdicción en el gobierno nacional), aún cuando ambas están reguladas por la Ley de Educación Superior (1995), modificada en 2015.

3. Se están implementando diferentes estrategias que posibiliten el cursado y la continuidad de los estudios: desde una política de becas de conectividad y de facilitación de acceso a recursos tecnológicos para aquellos sectores estudiantiles que veían limitadas sus posibilidades de conectividad, acceso a plataformas gratuitas, becas de ayuda económica y alimentaria, hasta repensar los cursos para atender a una población heterogénea, accesibilidad para estudiantes con capacidades diferentes, capacitación y acompañamiento a los profesores en el uso de tecnologías para garantizar la continuidad de los aprendizajes, entre otras.

4. Las políticas de internacionalización de la educación superior ocuparon un lugar relevante en la agenda de gobierno en el periodo analizado. En 2003 se crea el Programa de Internacionalización de la Educación Superior para la Cooperación Internacional (PIESCI), en el ámbito de la Secretaría de Políticas Universitarias, encargado de articular con instituciones universitarias estrategias y acciones de internacionalización como: becas, programas de intercambio académico, de promoción de las universidades, conformación de redes de investigación, acreditación regional de carreras de grado en el Mercosur Educativo, reconocimiento de títulos, entre otras numerosas acciones, etcétera. (Ver Larrea y Astur, 2012).

5. En el mismo periodo la participación en la educación superior de estudiantes del quintil más pobre creció más de $50 \%$ y la relación entre $20 \%$ más rico que cursa estudios de nivel superior y $20 \%$ más pobre pasó del $4 / 5$, a menos del 2/1. Argentina es el país con mayor equidad en el acceso a la educación superior: $18.5 \%$ provienen del primer quintil de ingresos y $24.3 \%$ de segundo quintil, lo que sumandos alcanzarían $43 \%$ del total de estudiantes. (Miranda, 2018; Del Bello, 2015; Pérez Rassetti, 2014).

6. El presupuesto por alumno creció $117 \%$ entre 2004 y 2010, con un leve aumento de la matrícula (3\%), mientras entre los años 2010-2018 el presupuesto creció en promedio $3 \%$, con años de caída y otros de crecimiento (Doberti, 2020, p. 20).

7. En el conurbano bonaerense, un territorio que concentra la mayor densidad población y los mayores índices de pobreza del país, se crearon ocho universidades nacionales: Universidad Nacional de Avellaneda (2009), Universidad Nacional de Moreno (2009), Universidad Nacional Arturo Jauretche (2009), Universidad Nacional de José C. Paz (2009), Universidad Nacional del Oeste (2010), Universidad Nacional de Hurlingham (2014), Universidad Scalabrini Ortiz (2015), Universidad Almirante Brown (2015).

8. Plan Estratégico de Formación de Ingenieros 2012-2016, como un instrumento para el logro de las metas de desarrollo para el dicho periodo.

9. Los Proyectos de Mejoramiento en la Enseñanza de carreras reguladas por el Estado (Art. $43^{\circ}$, Ley de Educación Superior) estaban destinados a financiar los compromisos asumidos en el plan de mejoras presentado por las unidades académicas que habían cumplimentado procesos de acreditación: Medicina (PROMED), Odontología 
(PROMOD), Veterinaria (PROMVET), Arquitectura (PROMARQ), Farmacia y Bioquímica (PROMFYB), Ingeniería Agronómica (PROMAGRO), Ingenierías (PROMEI I, II y PROMFORZ).

10. Durante la Presidencia de Cristina F. de Kirchner (2007-2015), se incorporaron otros programas de asignación especial a las universidades nacionales de carácter no competitivo: Programa de voluntariado universitario; Programa de internacionalización de la educación superior y cooperación internacional; Programa de promoción de la universidad argentina; Programa de Infraestructura universitaria; Programa de acciones complementarias a las becas bicentenario y de inclusión educativa (García de Fanelli, 2016).

11. Tomado del título del documental del Director Tristán Bauer donde narra la situación del país después de cuatro años de gobierno de la Alianza Cambiemos.

12. Tal es el caso del Programa PROGRESAR (creado en 2014), relanzado como plan de becas por el Gobierno de Cambiemos. En declaraciones al Diario La Nación, el ministro de Educación Alejandro Finocchiaro señalaba: "El Programa Progresar fue concebido casi como un subsidio, no había una lógica educativa”. En el futuro se pedirían mayores exigencias académicas y se premiaría el mérito y el compromiso. Las becas tendrían montos diferenciados según el nivel educativo, y para el caso de los universitarios se premiaría el rendimiento académico y la carrera seleccionada, privilegiando aquellas definidas como estratégicas en términos de desarrollo productivo y mercado de trabajo (Aliaga, 2018).

13. "Esa desarticulación se manifestó en una reacción en cadena de desmantelamientos de proyectos tecnológicos en: Fabricaciones Militares, la Agencia Nacional de Laboratorios Públicos, INVAP, Nucleoeléctrica Argentina, CNEA, ENSI, ARSAT, INTI, Río Turbio, SENASA, la Planta Industrial de Agua Pesada, INTA, Astilleros Río Santiago, entre otras instituciones y empresas públicas, que fue acompañada de la destrucción de grupos a través de despidos e incentivos a los retiros voluntarios de personal calificado y el desfinanciamiento sistémico creciente de las actividades de CYT" (Hurtado, 2019b, citado en Filmus, 2019). Argentina Innovadora 2020. Plan de Ciencia, Tecnología e Innovación Productiva (2013): https://www.argentina.gob.ar/sites/default/files/sintesis_2013_ai.pdf

14. Sobre las medidas más importantes del gobierno desde su asunción en diciembre de 2019: Plan Argentina contra el hambre; Ley de Solidaridad Social y reactivación productiva; IFE (Ingreso Familiar de Emergencia); Programa de Asistencia de Emergencia al trabajo y la Producción, entre otras numerosas medidas de gobierno en el marco de la Pandemia. Consultar: Ámbito Financiero, 11 de marzo de 2020. https://www.ambito.com/politica/alberto-fernandez/destaco-las-18-medidas-mas-importantes-su-primer-mes-gestion-n5076088

También consultar: https://www.argentina.gob.ar/coronavirus/medidas-gobierno

15. El Decreto Núm. 29.337 del 22 de noviembre de 1949 fundamenta la decisión en que "el Estado debe prestar todo su apoyo a los jóvenes estudiantes que aspiren a contribuir al bienestar y prosperidad de la Nación suprimiendo todo obstáculo que les impida o trabe el cumplimiento de tan notable como legítima vocación”. Vale señalar que la gratuidad de los estudios universitarios también fue uno de los reclamos de la Reforma de 1918. (Miranda, 2018; Miranda, 1993).

16. La economía argentina registra niveles de endeudamiento cercanos a $90 \%$ del PBI.

17. La CEPAL en el Informe especial Núm. 3 sobre la COVID-19, titulado El desafío social en tiempos de la COVID-19, analiza posibles escenarios sobre la evolución de la pobreza y de la extrema pobreza en América Latina y el Caribe a causa de la pandemia y llega a la conclusión que en todos los escenarios, la pobreza y la pobreza extrema aumentarían en todos los países. La pobreza en general aumentaría en Argentina, México, Ecuador y Brasil; los mayores aumentos de la pobreza extrema en México, Nicaragua y el Ecuador y la vulnerabilidad en las tres mayores economías de la región. Propone fortalecer el Estado de bienestar y la provisión universal de protección social para no caer en otra década perdida. (CEPAL, 2020, p. 3 y 19). 


\section{Referencias}

Aliaga, J. Panel. "La gratuidad para la comunidad universitaria”. Foro Internacional. A setenta años de la gratuidad de la educación superior universitaria. Universidad Tecnológica Nacional. Facultad Regional Buenos Aires, septiembre 2019.

Aliaga, J (2018). PROG.R.ES.AR será más difícil para los jóvenes. En Página personal, 17 de febrero de 2018. Consultado el 29 de mayo de 2020. Disponible en http://www.jorgealiaga.com.ar/?p=664

Anuario Estadístico 2005. Ministerio de Educación y Deportes de la Nación Secretaría de Innovación y Calidad Educativa. Dirección Nacional de Información y Estadística Educativa. Ciudad Autónoma de Buenos Aires. 2006.

Anuario 2015. Ministerio de Educación y Deportes de la Nación. Secretaría de Innovación y Calidad Educativa. Dirección Nacional de Información y Estadística Educativa. Ciudad Autónoma de Buenos Aires. 2016.

Anuario Estadístico Educativo 2018. Dirección de Información y Estadística Educativa. Ministro de Educación, Cultura, Ciencia y Tecnología de la Nación. Buenos Aires. 2019.

Astur, A. y Larrea, M. (2012). Políticas de internacionalización de la educación superior y cooperación internacional universitaria. PIESCI-Secretaría de Políticas Universitarias. Consultado el 27 de mayo de 2020. Disponible en: http://pep.unc.edu.ar/wp-content/uploads/sites/46/2017/02/ Pol\%C3\%ADticas-de internacionalizaci\%C3\%B3n-de-la-Eduaci\%C3\%B3n-Superior-Larrea-M-y-Astur-A.pdf

Ball, S. (2017). "Laboring to relate. Neoliberalism, embodied policy and network dinamics". Peabody Journal of Education, 92(1), pp. 29-41. doi: 10.1080/0161956X.2016.1264802

Ball, S. (2011). “¿Intelectuais ou técnicos? O papel indispensável da teoría nos estudos educacionais”. En Ball, S. y Mainardes, J. (orgs.). Políticas Educacionais. Questões e dilemas. Sao Paulo: Cortez Editora.

Bárcena, A. (2020). Presentación. Reunión "Hacia una nueva economía global: Encuentro universitario ministerial”. CELAC, México. Disponible en: https://www.cepal.org/sites/default/ files/presentation/files/final_finalcelac-28_mayo2020.pdf

Bauman, Z. "La única certeza es la incertidumbre”. El Periódico, 21/11/2008. Disponible en: https:// www.elperiodico.com/es/opinion/20081121/zygmunt-bauman-la-unica-certeza-es-la-incertidumbre-20844

CEPAL (2020) El desafío social en tiempos del Covid-19. Informe Especial Núm 3. Disponible en: https://repositorio.cepal.org/bitstream/handle/11362/45527/5/S2000325_es.pdf

Chiroleu, A. y Iazzetta, O. (2005). La reforma de la Educación Superior como capítulo de la reforma del Estado. En E. Rinessi, G. Soprano, y C. Suasnábar (Eds.), Universidad: Reformas y desafíos. Dilemas de la Educación Superior en Argentina y Brasil. Buenos Aires: Prometeo. pp. 15-38.

Consejo Interuniversitario Nacional (CIN) Declaración del Consejo de Rectores. 08/04/2020. https://www.cin.edu.ar/las-universidades-argentinas-frente-a-la-pandemia-del-covid-19/

Consejo Interuniversitario Nacional (CIN). Declaración del 83er Plenario de Rectoras y Rectores. Apoyo a la propuesta de reestructuración la deuda externa. 14/05/2020. https://www.cin.edu. ar/declaracion-apoyo-a-la-propuesta-de-reestructuracion-de-la-deuda-externa/

Conferencia Regional de Educación Superior de América Latina y el Caribe (CRES) 2018. IESALC-UNESCO-Universidad Nacional de Córdoba. Junio 2018.

Del Bello, J. "II Agenda de políticas universitarias”. En C. Marquis (Org.) La Agenda Universitaria II. Propuestas de Políticas Públicas. Buenos Aires: Ediciones Universidad de Palermo, 2015, p. 43-78.

Del Valle, D.; Montero, F. y Mauro, S. (Comp.) (2017). El derecho a la Universidad en perspectiva regional. Ciudad Autónoma de Buenos Aires: IEC-CONADU: CLACSO.

Dibbern, A. (2010). Presentación realizada en el v Taller Internacional "Las Políticas de Evaluación y Acreditación de la Calidad de la Educación Superior en América Latina y el Caribe: Una nueva agenda para la integración regional". La Habana, Cuba: IESALC.

Doberti, J.; Gabay, G. y Levy, M (2020). "El presupuesto universitario en Argentina: Cuánto, ¿cómo, dónde y a quiénes?”. En Cuadernos del inap Núm. 7. Buenos Aires: Instituto Nacional de Administración Pública. 
El Cronista. https://www.cronista.com/economiapolitica/La-industria-bajo-Cristina-y-Macrique-opinan-desde-el-sector-20190714-0020.html

Escudero, M. y Salto, D. (2019). "Más mercado, menos estado: el financiamiento de los posgrados en Argentina”, en Espacios en Blanco. Revista de Educación, 29 (1), pp. 65-84. Universidad Nacional de Tandil.

Fernández, A. Discurso de asunción a la Presidencia. 10/12/2019. Disponible en: https://www. casarosada.gob.ar/informacion/discursos/46596-palabras-del-presidente-alberto-fernandez-en-su-acto-de-asuncion-ante-la-asamblea-legislativa

Fernández, A. Discurso de apertura de las Sesiones Ordinarias del Congreso de la Nación. 01/03/2020. Disponible en: https://www.casarosada.gob.ar/informacion/discursos/46746-discurso-del-presidente-alberto-fernandez-al-encabezar-la-apertura-del-periodo-138-de-sesiones-ordinarias-del-congreso-de-la-nacion

Filmus, D. (2019). “La situación de la ciencia y tecnología en Argentina. Realidad y Desafíos”, en Análisis Carolina, Núm 21, Fundación Carolina, España. Consultado el 29 de mayo de 2020. Disponible en: https://www.fundacioncarolina.es/wp-content/uploads/2019/10/AC-21.pdf

Frente de Todos. Plataforma Electoral. Octubre 2019. https://frentedetodos.org/plataforma

García de Fanelli, A. (2016). Informe Nacional: Argentina. La Educación Superior en Iberoamerica. Sgo de Chile: cinda-Universia.

García de Fanelli, y A. Claverie, J. (2013). "Políticas públicas para la mejora de la calidad de las carreras de ingeniería: Estudio de caso del promei en la Argentina”, en Revista Argentina de Educación Superior. 5 (6): Disponible en: http://raes.org.ar/revistas/raes6_art1.pdf

García Delgado, D. y Gradin, A. (Comp.) (2017a). El neoliberalismo tardío. Teoría y Praxis. Documento Núm. 5. Buenos Aires: Flacso, 2017.

García Delgado, D. (2017b). "Encíclica Laudato Si' y modelos de desarrollo. La hermenéutica del rumbo”. En J.C. Scanonne Et al: Laudato Si'. Lecturas desde América Latina. Desarrollo, exclusión social y ecología integral. Buenos Aires: Fundación CICCUS.

Instituto de Estudios y Capacitación-Federación Nacional de Docentes Universitarios (IEC-CONADU) (2019). Análisis de la política de financiamiento para las Universidades Nacionales 2016. Primer semestre 2019. 15 Puntos básicos. Disponible en: https://iec.conadu.org.ar/files/publicaciones/1567090755_informe-2016-primer-semestre-2019-15-puntos.pdf

Instituto Internacional de la UNESCO para la Educación Superior en América Latina y el Caribe (IESAlC). Declaración y Plan de Acción de la Conferencia Regional de Educación Superior en América Latina y el Caribe 2008. UNESCO.

Observatorio Latinoamericano de la ciencia, la tecnología y la innovación de la OEI. Las universidades pilares básicos de la ciencia y tecnología en América Latina. Aportes a la CRES 2018. Buenos Aires, 2018.

Otero, A., Corica, A. y Merbilhaá, J. (2018). "Las universidades del conurbano bonaerense: influencias y contexto”, en Archivos de Ciencias de la Educación, 12 (14). https://doi.or$\mathrm{g} / 10.24215 / 23468866 \mathrm{e} 052$

La Nación. Qué esperan los científicos del próximo gobierno. Nora Bär. 9/12/2019. https://www. lanacion.com.ar/ciencia/que-esperan-cientificos-del-proximo-gobierno-nid2313890

Laval, C. (2018). Educación Superior en el siglo XXI: ¿bien público o mercancía?. Jornadas de Reflexión sobre tendencias en la educación superior. Uruguay: Universidad de la República, p.p. 39-58.

Ministerio de Educación, Ciencia y Tecnología. Secretaria de Políticas Universitarias. Proyecto de Mejoramiento de la Enseñanza en Ingeniería (PROMEI). Plan Plurianual 2005-2007. Buenos Aires: Subsecretaria de Gestión y Coordinación de Políticas Universitarias, 2005.

Ministerio de Educación, Ciencia y Tecnología. Secretaría de Políticas Universitarias. Programa de Calidad Universitaria. Proyecto de Mejoramiento de la Enseñanza en Ingeniería II (PROMEI II). Plan Plurianual 2007-2010. Buenos Aires: Subsecretaria de Gestión y Coordinación de Políticas Universitarias, mayo 2007.

Ministerio de Educación. Secretaria de Políticas Universitarias. Proyecto de Mejoramiento de la Enseñanza en Medicina (promed). Plan Plurianual 2011-2013. Buenos Aires: Subsecretaria de Gestión y Coordinación de Políticas Universitarias, 2010. 
Miranda, E. (1993). La Formación del Sistema Universitario Nacional. Desarrollo y Crisis 1880-1946. Dirección General de Publicaciones, Universidad Nacional de Córdoba.

Miranda, E. (2013). "De la selección a la universalización. Los desafíos de la obligatoriedad de la educación secundaria”. En Espacios en Blanco, Revista de Educación, (23), p.p 9-32. Universidad Nacional de Tandil.

Miranda, E. (Coord.) (2014). Democratización de la Educación Superior. Una mirada desde el MERCOSUR. Córdoba: Narvaja Editor. Consultado 20/05/2020 Disponible: http://nemercosur.siu.edu.ar/ documentos/Democratizacion_de_la_Educacion_Superior.pdf

Miranda, E. (2018). "El derecho a la universidad en Argentina. Una mirada desde la igualdad y el reconocimiento del otro". En Sisyphus. Journal of Education, 6 (1), p.p. 79-96. Consultado 29/05/2020. Disponible en: http://revistas.rcaap.pt/sisyphus/issue/view/816

Miranda, E. (2020). "A política educativa como negócio: Ajuste do orçamento, discurso meritocrático e o " "davos” ” da educação na Argentina” (2015-2019), en Revista Espaço Pedagógico, 27 (1), p.p. 9-29, 1 fev. 2020. doi: https://doi.org/10.5335/rep.v27i1.10572

Morduchowicz, A. (2019). "El financiamiento educativo argentino", en Propuesta Educativa, Año 28, núm. 52, noviembre 2019, pp. 11 a 23. Flacso-Buenos Aires.

Morosini, M. (2014) “Qualidade da Educação Superior e Contextos Emergentes”, en Avaliação, 19 (2), julio, p.p. 385-404, Campinas, Sorocaba. São Paulo.

Pérez Rasetti, C. (2004). La acreditación y la formación de los ingenieros en la Argentina. Buenos Aires: CONEAU.

Pérez Rasetti, C. (2014). "La expansión de la educación universitaria en Argentina: políticas y lógicas”, en Integración y Conocimiento. Revista del Núcleo de Estudios e Investigaciones en Educación Superior del MERCOSUR, (2), p.p. 8-32. Consultado 29/05/2020. Disponible en: http:// revistas.unc.edu.ar/index.php/integracionyconocimiento/issue/current

Red/Acción. 27/10/2019. Disponible en https://www.redaccion.com.ar/educacion-que-politicas-impulsaria-alberto-fernandez-durante-su-gestion/

Sistema de Información de Tendencias Educativas en América Latina (SITEAL) (2015). Indicadores Estadísticos. IIPE. Buenos Aires. Consultado el 20 de mayo de 2020. Disponible en: https:// www.siteal.iiep.unesco.org/indicadores

Stefani, F. (2018). El rol actual y futuro de la ciencia en la innovación industrial y el crecimiento económico en Argentina. s/d. Consultado el 29 de mayo de 2020. Disponible en: https://www. biologia.org.ar/wp-content/uploads/2018/10/Stefani-2018_Rol-actual-y-futuro-de-la-ciencia_en-la-innovacion-industrial-en-Argentina.pdf

Suasnábar, C. y Rovelli, L. (2012). "Impensar las políticas universitarias en la Argentina reciente”. En Chiroleu, A.; Marquina, A. y Rinesi, E. (Comp.). La política universitaria de los gobiernos de Kirchner. Continuidades, rupturas, complejidades. Los Polvorines: Universidad Nacional de General Sarmiento.

Tenti Fanfani, E (1994). "Del intelectual orgánico al analista simbólico”, en Revista de Ciencias Sociales, (1), p.p. 19-29. Disponible en RIDAA-UNQ. Repositorio Institucional Digital de Acceso Abierto de la Universidad Nacional de Quilmes. Consultado 29/05/2020. Disponible en: https://ridaa.unq.edu.ar/bitstream/handle/20.500.11807/1287/04_RCS_1994_n1_articulos2.pdf?sequence $=1 \&$ isAllowed=y

Quevedo, L. A. (2017). “América Latina en movimiento”. Prólogo. En D. García Delgado y A. Gradin. El Neoliberalismo tardío. Teoría y Praxis. Documento de Trabajo Núm. 5. Buenos Aires: Flacso. Disponible en: https://www.flacso.org.ar/wp-content/uploads/2017/07/El-Neoliberalismo-tardio-Teoria-y-Praxis.pdf

Verger, A., Curran, M. y Parcerisa, Ll. (2015). "La trayectoria de una reforma educativa global: el caso de la nueva gestión pública en el sistema educativo catalán”, en Educação \& Sociedade, 36 (132), pp.675-697.

Yelicich, C. (2017). “Aproximaciones al análisis epistemológico de la Nueva Gestión Pública”. En Revista de Estudios Teóricos y Epistemológicos en Política Educativa, vol. 2, pp.1-17. http:// dx.doi.org/10.5212/retepe.v.2.013. Disponible en http://www.revistas2.uepg.br/index.php/ retepe/article/view/10544 